\title{
Clinical Significance of ST Segment Depression in Lead aVR to Predict Culprit Artery in An Acute Inferior Wall Myocardial Infarction.
}

Madhu Gupta ${ }^{1}$, Maheswar Prasad Kurmi ${ }^{2}$, Bhoj Raj Sharma ${ }^{1}$, Liping Chen ${ }^{1}$, Ravi Shahi ${ }^{1}$, , Sun Jian ${ }^{1}$

${ }^{I}$ Department of Cardiovascular Diseases, Norman Bethune College of Medicine,

The First Hospital of Jilin University, PR China.

${ }^{2}$ Department of Medicine, National Medical College, Nepal.

\section{Citation}

Madhu Gupta, Bhoj Raj Sharma, Liping Chen et al. Clinical Significance of ST Segment Depression in Lead aVR to Predict Culprit Artery in An Acute Inferior Wall Myocardial Infarction. NepaleseHeartJournal2015;12(1):5-9

\section{Key words}

Inferior wall MI, Lead aVR, ST Depression

\section{INTRODUCTION}

The inferior wall (I-AMI) and inferolateral wall of the left ventricle are supplied by the Right Coronary Artery (RCA) and Left circumflex LCx. An I-AMI is caused by an occlusion of either the RCA or LCx, and predicting the artery involved can be challenging because the dominance of the RCA and LCx can vary significantly among

\begin{abstract}
Background and Aims: The main objective of this study is to analyze between left circumflex artery and right coronary artery as a presumptive predictor of a culprit artery in patients with an acute inferior wall ST elevation myocardial infarction according to ST segment change in lead aVR.
\end{abstract}

Methods: This study included 145 consecutive patients who presented with an inferior wall ST elevation myocardial infarction and underwent coronary angiography. Clinical and angiographic findings were compared between patients with and without aVR depression $\geq 0.1 \mathrm{mV}$.

Results: The sensitivity and specificity of ST segment depression in lead aVR to predict left circumflex artery as the culprit artery were 69\% and $85 \%$, respectively, and the positive predictive value and negative predictive value to predict left circumflex artery as the culprit arteries were $66 \%$ and $87 \%$, respectively.

Conclusion: Our study supports, the presence of ST segment depression in lead aVR is associated with good specificity and negative predictive value and modest sensitivity and positive predictive value to determine left circumflex artery as an infarct related artery in an acute ST segment elevation inferior wall myocardial infarction.

\section{Corresponding Author}

\section{Sun Jian}

Department of Cardiovascular Diseases Norman Bethune College of Medicine,

The First Hospital of Jilin University, Jinlin, PR China Email: sunjian418@gmail.com 
patients. Multiple ECG criteria have been studied to predict the culprit artery based on ST segment elevation and ST segment depression in different leads.1-9 Many recent studies have been suggesting ST depression in lead aVR as a predictor of LCx artery occlusion.10-12 The purpose of this study is to examine whether the culprit artery in case of the I-AMI can be predicted by lead aVR depression and also to study its sensitivity, specificity and predictive values.

\section{METHODS}

In order to study retrospectively, we collected 312 patients having an acute I-AMI, admitted in coronary care unit of first hospital of Norman Bethune college of Medicine from $1^{\text {st }}$ January 2013 to $31^{\text {st }}$ December 2013 and who subsequently underwent coronary angiography during hospitalization. Only 145 patients (male 105 and female 40) met our inclusion criteria, which included chest pain lasting for $\geq 30$ minutes before hospital admission, an increased in serum creatinine kinase $(\mathrm{CK}-\mathrm{MB})$ greater than twice the upper limit, ECG abnormalities that included ST segment elevation $>0.1 \mathrm{mV}(1 \mathrm{~mm})$ in at least 2 or 3 inferior leads (II, III, aVF), ST segment depression of at least $0.1 \mathrm{mV}$, and coronary angiography showing total occlusion or critical stenosis $>70 \%$ in a single vessel either LCx or RCA. Patients with previous history of an acute myocardial infarction, coronary artery bypass surgery or percutaneous coronary intervention prior to current hospitalization, evidence of recent left bundle branch block, and significant stenosis in both LCx and RCA or triple vessel disease were excluded. A 12 lead ECG was recorded in all patients. The ST segment deviation was measured manually to the nearest $0.5 \mathrm{~mm}, 80 \mathrm{~ms}$ after the $\mathrm{J}$ point by a single cardiologist who was unaware of all clinical and angiographic findings. ST segment depression in lead aVR of at least $0.1 \mathrm{mv}(1 \mathrm{~mm})$ was the most important criterion to distinguish between LCx and RCA as presumptive predictor of the culprit artery. Coronary angiography was performed during hospitalization in all patients. The results of this test were compared with those obtained from ECG to distinguish the real culprit artery. All patients underwent PCI following coronary angiography. Data were expressed as the mean $\pm \mathrm{SD}$ for continuous variables and percentages for categorical variables. Statistical significance $(p<0.05)$ was assessed by the chi - square test or Fisher exact test for categorical variables and Mann-Whitney test for numerical variables. The sensitivity, specificity, positive predictive value and negative predictive value were evaluated in the two coronary arteries.

\section{RESULTS}

A total of 145 patients (male 105 and female 40) were included in the study, aged 30 to 76 years (mean age $58 \pm 11$ ). Overall, 44 patients $(30 \%)$ were found to have aVR depression and 101 patients $(70 \%)$ were without aVR depression. The culprit artery was found to be the right coronary artery in 103 patients $(71 \%)$, the LC in 42 patients $(29 \%)$. Baseline clinical characteristics were similar between the 2 groups (Table. 1). The LCx disease was found significantly

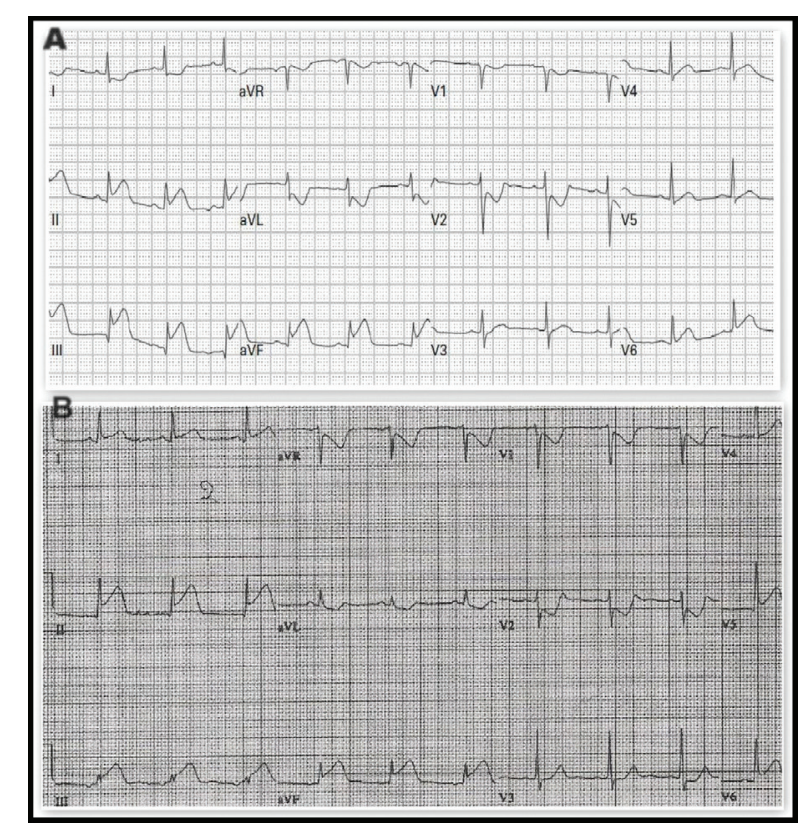

Figure 1. (A) A 65 years old male with ECG showing changes of acute inferior wall MI caused by occlusion of the RCA;ST segment depression in lead aVR $<1 \mathrm{~mm}$, the magnitude of ST segment elevation in lead III>II,ST depression in lead I; ST segment isoelectric in lead V1 with depression in V2, strongly suggest acute RV. (B) A 59 years old man with ECG showing changes of acute inferior wall MI caused by occlusion of the left circumflex artery; ST segment depression in lead aVR $>1 \mathrm{~mm}$,ST elevation in lead II >III;ST segment elevation in lead I; ST depression in leads V1-V2 suggesting acute posterior injury. 
Table 1 : General clinical characteristics

\begin{tabular}{|l|c|c|c|}
\hline \multicolumn{1}{|c|}{ Variable } & $\begin{array}{c}\text { Patients } \\
\text { with ST } \\
\text { depression } \\
\text { in aVR } \\
\mathrm{n}=44\end{array}$ & $\begin{array}{c}\text { Patients } \\
\text { without ST } \\
\text { depression } \\
\text { in aVR } \\
\mathrm{n}=101\end{array}$ & \\
\hline \hline Age & $58 \pm 11$ & $62 \pm 15$ & 0.476 \\
\hline Male & $32(73)$ & $73(72)$ & 0.956 \\
\hline Diabetes mellitus & $18(41)$ & $21(21)$ & 0.012 \\
\hline Smoking & $18(41)$ & $45(44)$ & 0.684 \\
\hline Dyslipidemia & $20(45)$ & $72(71)$ & 0.003 \\
\hline Hypertension & $22(50)$ & $48(48)$ & 0.784 \\
\hline Culprit artery & & & 0.0001 \\
\hline LCX & $29(66)$ & $13(13)$ & \\
\hline RCA & $15(34)$ & $88(87)$ & \\
\hline Killip class I & $31(70)$ & $81(80)$ & 0.198 \\
\hline CK-MB (ng/ml) & $227 \pm 73$ & $136 \pm 67$ & 0.367 \\
\hline LVEF (\%) & $57 \pm 90$ & $59 \pm 70$ & 0.51729 \\
\hline TIMI 0 & $17(39)$ & $26(26)$ & 0.118 \\
\hline
\end{tabular}

higher proportion in ST depression in lead aVR (n-29; 66\%) than without ST depression in lead aVR ( $\mathrm{n}-13 ; 13 \%)(\mathrm{p}=0.0001)$. The sensitivity and specificity of ST-segment depression in lead aVR for LCx as the culprit artery were $69 \%$ and $85 \%$ respectively. The positive predictive and negative predictive value for $\mathrm{LC}$ as the culprit arteries were $66 \%$ and $87 \%$. However, 101 patients, with ST segment elevation or ST-segment isoelectric or ST segment depression $<1 \mathrm{~mm}$ in the lead aVR, classified as patients without depression in lead aVR were significant for RCA as the culprit artery in $88(87 \%)$ compared to $15(34 \%)$ (p-0001) patients. The sensitivity, specificity, positive predictive value and negative predictive value for RCA as the culprit artery were $85 \%, 69 \%, 87 \%$ and $66 \%$ respectively.

\section{DISCUSSION}

In an I-AMI, the prognosis of patients depends on identification of the occluded artery (RCA or LCx). Patients with occlusion in the RCA (approx. 80\% of cases) is frequently associated with right ventricle involvement and have a poorer
Table 2 : The sensitivity and specificity of ECG criteria to predict culprit artery

\begin{tabular}{|l|l|l|l|l|}
\hline Vessel & $\begin{array}{c}\text { Sensitivity } \\
(\%)\end{array}$ & $\begin{array}{c}\text { Specificity } \\
(\%)\end{array}$ & $\begin{array}{l}\text { Positive } \\
\text { predictive } \\
\text { value (\%) }\end{array}$ & $\begin{array}{l}\text { Negative } \\
\text { predictive } \\
\text { value (\%) }\end{array}$ \\
\hline LCX & 69 & 85 & 66 & 87 \\
\hline RCA & 85 & 69 & 87 & 66 \\
\hline
\end{tabular}

outcome $\mathrm{e}^{1 ; 13-14}$. The identification of arteries and the size of infarct plays an important role in guiding the reperfusion therapy. The display of lead aVR $\left(-150^{\circ}\right)$ in an inverted format as lead $-\mathrm{aVR}\left(+30^{\circ}\right)$ lies between lead I $\left(0^{\circ}\right)$ and lead II $\left(60^{\circ}\right)$. Thus, aVR depression means $-\mathrm{aVR}$ elevation, which represents the infarct of the apical and inferolateral walls, usually supplied by the posterolateral branch of either the RCA or LCx itself. ${ }^{10}$ The aVR depression suggests the involvement of LCX or a large RCA with a large posterolateral branch occlusion. This finding is consistent with the observation that $60 \%$ of both dominant RCA and dominant LCx infarctions had aVR depression. ${ }^{15}$

Several studies have considered the clinical importance of ST-segment depression in lead aVR in an I-AMI to identify the culprit artery. Tieralaet al, in the HAAMU trial, a non-

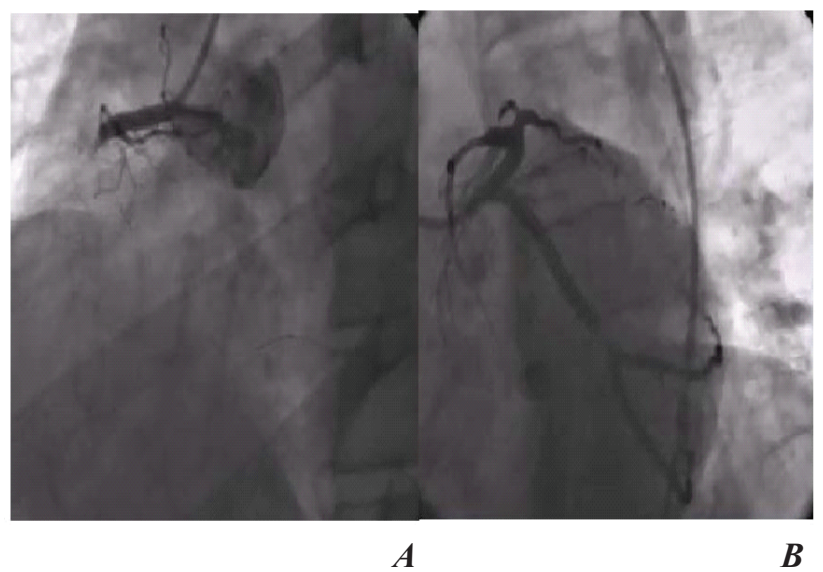

Figure 2.

A Coronary angiogram showed RCA occlusion. $B$ Coronary angiogram showed critical stenosis of $L C x$ 
randomized prospective study of an acute I-AMI, proposed a new algorithm and compared it with the prior algorithm from Fiol et al to predict the culprit artery. Among 98 patients included in their study, they found with ECG and angiography a positive and negative predictive value for the prediction of LCx or RCA as an IRA of $75 \%$ and $94 \%$, $92 \%$ and $75 \%$ respectively. The sensitivity and specificity fortheRCA, 96\% and $56 \%$,respectively, compared to that of Fiol et al with $96 \%$ and $38 \%$ respectively. Whereas, for the $\mathrm{LCx}$, the sensitivity and specificity were $64 \%$ and $96 \%$, respectively, compared to that of Fiol et al with $42 \%$ and $96 \%$. They concluded that ST depression in lead aVR is better for predicting LCx as the culprit artery. ${ }^{8-9}$ Kanei et al, with the main aim to review all algorithms and compare them to ST depression in the lead aVR to predict the culprit artery in an I-AMI found the sensitivity and specificity of ST segment depression in lead aVR to predict the LCX as a culprit artery were $53 \%$ and $83 \%$ respectively. RCA as the culprit artery, the sensitivity and specificity were, $86 \%$ and $55 \%$, respectively. ${ }^{16}$

Similarly, Sun et al, investigated the sensitivity and specificity of aVR in differentiating LCx form RCA as the culprit artery for an I-AMI. The sensitivity and specificity of LCx were 70\% and $94 \%$, respectively ${ }^{12}$ and concluded that ST depression in lead aVR is associated with a higher specificity and a good sensitivity for LCx lesion, and all patients who are suspected of having an I-AMI should be carefully examined for aVR lead.

The aim of our study was to make the differential diagnosis, in 12 leads ECG, between LCx and RCA as a presumptive predictor of a culprit artery in patients with an I-AMI according to ST changes in lead aVR. ST depression in lead aVR is a simple, efficient method that does not require multiple complicated steps. ST segment depression in lead aVR was associated with LCx involvement with sensitivity, specificity, positive, and negative predictive values of $69 \%, 85 \%, 66 \%$ and $87 \%$, respectively. The sensitivity found in our study was higher, in comparison to the following studies; Kanei et al (53\%), Tierala et al (64\%), Fiol et al (42\%). However, the specificity (85\%) was less than the Tierala et al and Fiol et al but more than Kanei et al.

Conversely, 101 patients without ST depression in lead aVR were significantly associated with a culprit RCA, 88 (87\%) compared to 15 (34\%) and corresponding to the sensitivity, specificity, positive, and negative predictive value of $85 \%, 69 \%, 87 \%$ and $66 \%$, respectively. From our results, the sensitivity obtained to predict RCA as a culprit artery was slightly lower than those found in Kanei et al $(86 \%)$, Tierala et al (96\%), Fiol et al (96\%), whereas the specificity were higher than found in the above studies. Those differences in the sensitivity, specificity and predictive value in the previous and our studies, can be explained by the fact that there are some variations in the used criteria, sample size, delay in ECG recording and angiography.

The present study had several limitations that need to be considered when interpreting the clinical implications of our findings. This was a small sample size, retrospective study in a single center. The study lacked the follow-up and complications like reinfarction, post infarction angina, arrhythmias and mortality and the evaluation of right precordial leads (V4R) that have further helped to identify the culprit artery in an I-AMI.

\section{CONCLUSION}

Our study supports, the presence of ST segment depression in lead aVR is associated with good specificity and negative predictive value and modest sensitivity and positive predictive value to determine $\mathrm{LCx}$ as a culprit vessel in an I-AMI . Thus, the use of the ST segment in lead aVR remains a simple method with good result for predicting culprit artery. 


\section{REFERENCES}

1. Kosuge M, Kimura K, Ishikawa T, et al. New electrocardiographies criteria for predicting the site of coronary artery occlusion in inferior wall acute myocardial infarction.

Am J Cardiol 1998;82:1318-22.

2. Chia BL, Yip JW, Tan HC. Usefulness of ST elevation II/III ratio and ST deviation in lead I for identifying the culprit artery in inferior wall acute myocardial infarction. Am J Cardiol 2000;86:341-3.

3. Herz I, Assali AR, Adler T, et al. New electrocardiographic criteria for predicting either the right or left circumflex artery as the culprit coronary artery in inferior wall acute myocardial infarction. Am J Cardiol 1997;80:1343-5.

4. Zimetbaum PJ, Krishnan S, Gold A, et al. Usefulness of ST-segment elevation in lead III exceeding that of lead II for identifying the location of the totally occluded coronary artery in inferior wall myocardial infarction. Am J Cardiol 1998;81:918-9

5. Bairey CN, Shah PK, Lew AS, et al. Electrocardiographic differentiation of occlusion of the circumflex versus the right coronary artery as a cause of inferior acute myocardial infarction. Am J Cardiol 1987;60:456-9.

6. Hasdai D, Birnbaum Y, Herz I, et al. ST segment depression in lateral limb leads in inferior wall acute myocardial infarction. Eur Heart J 1995;16:1549-53.

7. Assali AR, Herz I, Vaturi M, et al. Electrocardiographic criteria for predicting the culprit artery in inferior wall acute myocardial infarction. Am J Cardiol 1999;84:87-9.

8. Fiol M, Cygankiewicz I, Carrillo A, et al. Value of electrocardiographic algorithm based on "ups and downs" of ST in assessment of a culprit artery in evolving inferior wall acute myocardial infarction. Am J Cardiol 2004;94(6):709-14.

9. Tierala I, Nikus KC, Sclarovsky S, et al. Predicting the culprit artery in acute ST-elevation myocardial infarction and introducing a new algorithm to predict infarct-related artery in inferior ST-elevation myocardial infarction: correlation with coronary anatomy in the HAAMU Trial. J Electrocardiol 2009;42:120.

10. Menown IBA, Adgey AAJ. Improving the ECG classification of inferior and lateral myocardial infarction by inversion of lead aVR.Heart 2000; 83(6):657-60

11. Nair B, Glancy L. ECG Discrimination between right and left circumflex coronary arterial occlusion in patients with acute inferior myocardial infarction. Chest 2002;122:134-39

12. Sun TW, Wang LX, Zhang YZ, et al. The value of ECG leads aVR in the differential diagnosis of acute inferior wall myocardial infarction. Intern Med 2007; 46:795-9.

13. Berger PB, Ryan TJ. Inferior myocardial infarction: high risk subgroups. Circulation 1990;81:401-411.

14. Zehender M, Kasper W, Kauder E, et al. Right ventricular infarction as an independent predictor of prognosis after acute inferior infarction. N Engl J Med 1993;328:981-988.

15. Zhan ZQ, Wang W, Dang SY, et al. Electrocardiographic characteristics in angiographically documented occlusion of the dominant left circumflex artery with acute inferior myocardial infarction: limitations of ST elevation III/II ratio and ST deviation in lateral limb leads. J Electrocardiol 2009;42(5):432-9.

16. Yumiko Kanei, Jyoti Sharma, Ravi Diwan, et al. ST- segment depression in aVR as a predictor of culprit artery and infarct size in acute inferior wall ST-segment elevation myocardial infarction. Journal of Electrocardiography 2010;43: 132-135. 